\title{
The apoB100/apoAl ratio is independently associated with the severity of coronary heart disease: a cross sectional study in patients undergoing coronary angiography
}

Yongyan Song ${ }^{1}$, Yang Yang ${ }^{2}$, Jingxiao Zhang ${ }^{3}$, Yanmei Wang ${ }^{3,4}$, Wenfeng $\mathrm{He}^{3}$, Xiaoming Zhang ${ }^{5}$, Jie Zhu ${ }^{2}$ and Zhan $\mathrm{Lu}^{3^{*}}$ (D)

\begin{abstract}
Background: Lipoprotein ratios have been shown to be associated with the occurrence of coronary heart disease (CHD), but little is known about their relationships with the severity of CHD.

Methods: A total of 792 angiographically defined CHD patients were enrolled following their admission. Patients were stratified into three groups based on the tertile of the Gensini scores $\left(\leq 33^{\text {rd }}\right.$ percentile, $33^{\text {rd }}$ to $66^{\text {th }}$ percentile and $\geq 66^{\text {th }}$ percentile) or the number of stenotic coronary branches (single-branch stenosis, double-branch stenosis and multi-branch stenosis). Demographic and biochemical data were collected and lipoprotein ratios were calculated. Logistic regression and path analysis were employed to examine the relationships between the lipoprotein ratios and the severity of CHD.

Results: The ratios of low-density lipoprotein cholesterol (LDL-C)/high-density lipoprotein cholesterol (HDL-C) and apolipoprotein B100 (apoB100)/apolipoprotein Al (apoAl) increased with the tertile of the Gensini scores $(P<0.05$ for both). The ratios of triglyceride (TG)/HDL-C, total cholesterol (TC)/HDL-C, LDL-C/HDL-C and apoB100/apoAl increased with the number of stenotic coronary branches $(P<0.05$ for all). The univariate logistic regression showed that the ratios of TC/HDL-C, LDL-C/HDL-C and apoB100/apoAl were positively associated with both the tertile of the Gensini scores and the number of stenotic vessels $(P<0.05$ for all), and the ratio of TG/HDL-C was positively associated with the number of stenotic vessels $(P<0.05)$. In multivariate logistic analysis, only the ratio of apoB100/ apoAl was independently and positively associated with the tertile of the Gensini scores (OR=2.93, $95 \% \mathrm{Cl}=$ 1.17-7.34, $P=0.022)$ and the number of stenotic vessels $(\mathrm{OR}=3.14,95 \% \mathrm{Cl}=1.01-6.47, P=0.048)$ after adjusting for the possible confounding variables. The apoB100/apoAl ratio was also shown to be a direct mediator between the risk factors including age, BMI, HDL-C, LDL-C, apoB100 and apoAl and the severity of CHD by path analysis.
\end{abstract}

Conclusion: Our data indicate that the apoB100/apoAl ratio could be a useful predictor for evaluating the severity of coronary stenosis in CHD patients.

Keywords: Lipoprotein ratio, ApoB100/apoAl, Coronary heart disease, Severity, Gensini score

\footnotetext{
* Correspondence: 346627249@qq.com

${ }^{3}$ Department of Cardiology, Affiliated Hospital of North Sichuan Medical

College, Nanchong 637000, Sichuan Province, P. R. China

Full list of author information is available at the end of the article
} 


\section{Background}

Coronary heart disease (CHD) is the major cause of death in developed countries and some developing countries like China [1]. CHD is recognized as a multifactorial disease, and dyslipidemia is closely associated with the progression of coronary atherosclerosis. Yusuf $S$ and colleagues [2] demonstrated that dyslipidemia could account for around $50 \%$ of the population-attributable risk for CHD. Reliable indexes for assessing the CHD risk and the extent of coronary stenosis are pivotal to manage and prevent this critical disease. The roles of plasma low-density lipoprotein cholesterol (LDL-C), total cholesterol (TC), highdensity lipoprotein cholesterol (HDL-C) and triglycerides (TG) in the development of CHD have been well established. Currently, increases in LDL-C, TC, TG and/or decreases in HDL-C are widely used to evaluate the risk of $\mathrm{CHD}$ in clinical practice. According to the 2013 ACC/AHA blood cholesterol guidelines [3] and the Adult Treatment Panel III (ATP III) Guidelines [4] of the United States, LDL-C is considered as the major cause of CHD and used as the primary target for therapy, and the other lipid parameters were used as the secondary or supplementary targets.

Although there were growing arguments that the lipoprotein ratios, which have atherogenic components (TG, TC and LDL-C) in the numerator and antiatherogenic components (HDL-C) in the denominator, could be better predictors for the occurrence [5-7] and development of CHD [8-12] than the individual lipid parameters, the roles of the lipoprotein ratios in the pathogenesis of CHD are still obscure. One point to support these arguments is that the lipoprotein ratios can comprehensively reflect the balance between the atherogenic and antiatherogenic potentials in one individual. An increase in TG, TC or LDL-C concentrations is an atherogenic lipid marker, whereas reduced HDL-C concentration is an antiatherogenic trait which may also be correlated with numerous other cardiovascular risk factors, including the components of the metabolic syndrome. In an angiography-based study, Lippi $U$ et al. [8] were the first to report that TC/HDL-C and LDL-C/HDL-C are statistically related to the severity of CHD. Their findings were later proved by another study [9] from Zhejiang University, China, in which the researchers demonstrated that the ratios of LDL-C/HDL-C and TC/HDL-C were significantly and positively correlated with the coronary artery lesions. A recent crosssectional association study [10] in a Lebanese population further revealed that the TC/HDL-C ratio $\geq 4$ significantly predicts $\geq 50 \%$ stenosis in all vessels. The ratio of TG/HDL-C was also found to be associated with the severity of CHD in two studies from
Ruijin Hospital, Shanghai Jiaotong University, China [11] and Insituto do Coração, Universidade de São Paulo, Brazil [12], respectively.

However, none of these studies on outcomes have evaluated the association of the apolipoprotein B100 (apoB100)/apolipoprotein AI (apoAI) ratio with the severity of $\mathrm{CHD}$. In theory, the apoB100/apoAI ratio should be an even better predictor for evaluating the severity of CHD than the HDL-C related ratios. Each particle of the atherogenic lipoproteins [low-density lipoprotein, very low-density lipoprotein, intermediary density lipoprotein and lipoprotein (a)] carries one apoB100 molecule, so the concentrations of plasma apoB100 can reflect the total number of atherogenic particles. On the other hand, apoAI accounts for about $70 \%$ of the total apolipoproteins in high-density lipoprotein; the plasma content of apoAI represents the total of antiatherogenic particles. Therefore, the apoB100/apoAI ratio might be a superior marker for CHD development in that it can comprehensively reflect the balance between the atherogenic particles and antiatherogenic particles. Several studies [13-16] have suggested that the apoB100/apoAI ratio is better than the HDL-C related ratios in predicting the occurrence of $\mathrm{CHD}$. In a prospective apolipoprotein-related mortality risk (AMORIS) study [13], the researchers reported that the apoB100/ apoAI ratio was strongly related to increased coronary risk, and was superior to any of the HDL-C related ratios in predicting CHD in a large sample of 69,030 men and 57,168 women above 40 years of age. Liem AH et al. [14] demonstrated that the apoB100/apoAI, but not the LDL-C/HDL-C ratio, is positively associated with cardiovascular events in statin-treated patients with known CHD.

We previously demonstrated that the lipoprotein ratios are superior to the individual lipid parameters as predictors for $\mathrm{CHD}$ occurrence, and the apoB100/apoAI ratio is the best one to predict CHD [7]. In the current study, we further investigated the associations between the ratios of TG/HDL-C, TC/HDL-C, LDL-C/HDL-C and apoB100/apoAI and the severity of CHD. We hypothesized that higher levels of TG/HDL-C, TC/HDL-C, LDL-C/HDL-C, and especially apoB100/apoAI are associated with more severe CHD.

\section{Results}

\section{Clinical characteristics of the study population}

The CHD patients were divided into three groups according to the tertile of the Gensini scores: group 1: $\leq$ $33^{\text {rd }}$ percentile (scores $\leq 11.5$ ); group 2: $33^{\text {rd }}$ to $66^{\text {th }}$ percentile (scores 12-32); group 3: $\geq 66^{\text {th }}$ percentile (scores $\geq 33$ ). The anthropometric and biochemical characteristics of the three groups are shown in Table 1. The mean age, and the prevalence of male gender and smoking increased with the tertile of the Gensini scores $(P<$ 
Table 1 Clinical characteristics of the CHD patients according to the tertile of the Gensini scores

\begin{tabular}{|c|c|c|c|c|}
\hline \multirow[t]{2}{*}{ Variables } & Group 1 & Group 2 & Group 3 & \multirow[t]{2}{*}{$P$ value } \\
\hline & $\leq 33^{\text {rd }}$ percentile $(n=265)$ & $33^{\text {rd }}$ to $66^{\text {th }}$ percentile $(n=261)$ & $\geq 66^{\text {th }}$ percentile $(n=266)$ & \\
\hline \multicolumn{5}{|l|}{ Non-lipid variables } \\
\hline Age, years & $62.06 \pm 9.98$ & $62.68 \pm 10.61$ & $64.95 \pm 9.67$ & 0.003 \\
\hline Male gender, n (\%) & $171(64.5)$ & $172(65.9)$ & $204(76.7)$ & 0.004 \\
\hline Weight, kg & $64.95 \pm 9.29$ & $63.39 \pm 9.36$ & $64.67 \pm 9.63$ & 0.225 \\
\hline $\mathrm{BMl}, \mathrm{kg} / \mathrm{m}^{2}$ & $24.56 \pm 2.84$ & $24.15 \pm 3.03$ & $24.16 \pm 3.04$ & 0.399 \\
\hline Smokers, n (\%) & 95 (35.8) & $120(46.0)$ & $133(50.0)$ & 0.003 \\
\hline Hypertension, n (\%) & $119(44.9)$ & $141(54.0)$ & $138(51.9)$ & 0.091 \\
\hline Diabetes, n (\%) & $35(13.2)$ & $34(13.0)$ & $51(19.2)$ & 0.080 \\
\hline \multicolumn{5}{|l|}{ Individual lipid variables } \\
\hline $\mathrm{TG}, \mathrm{mmol} / \mathrm{L}$ & $1.53 \pm 0.90$ & $1.49 \pm 1.09$ & $1.66 \pm 1.14$ & 0.156 \\
\hline $\mathrm{TC}, \mathrm{mmol} / \mathrm{L}$ & $4.16 \pm 0.94$ & $4.22 \pm 1.01$ & $4.23 \pm 1.20$ & 0.728 \\
\hline $\mathrm{HDL}-\mathrm{C}, \mathrm{mmol} / \mathrm{L}$ & $1.01 \pm 0.29$ & $1.02 \pm 0.34$ & $0.93 \pm 0.29$ & 0.001 \\
\hline $\mathrm{LDL}-\mathrm{C}, \mathrm{mmol} / \mathrm{L}$ & $2.37 \pm 0.75$ & $2.44 \pm 0.81$ & $2.49 \pm 0.94$ & 0.263 \\
\hline ApoAl, g/L & $1.03 \pm 0.19$ & $1.02 \pm 0.21$ & $0.95 \pm 0.21$ & $<0.001$ \\
\hline ApoB100, g/L & $0.76 \pm 0.24$ & $0.77 \pm 0.25$ & $0.80 \pm 0.28$ & 0.204 \\
\hline \multicolumn{5}{|l|}{ Lipoprotein ratios } \\
\hline $\mathrm{TG} / \mathrm{HDL}-\mathrm{C}$ & $1.83 \pm 2.06$ & $2.09 \pm 4.41$ & $2.16 \pm 2.53$ & 0.466 \\
\hline $\mathrm{TC} / \mathrm{HDL}-\mathrm{C}$ & $4.51 \pm 1.92$ & $4.73 \pm 3.58$ & $5.03 \pm 3.18$ & 0.079 \\
\hline LDL-C/HDL-C & $2.57 \pm 1.11$ & $2.70 \pm 1.75$ & $2.96 \pm 2.01$ & 0.024 \\
\hline ApoB/apoAl & $0.76 \pm 0.27$ & $0.78 \pm 0.29$ & $0.88 \pm 0.43$ & $<0.001$ \\
\hline
\end{tabular}

The differences across the tertile of the Gensini scores were calculated by Chi-square test for categorical variables, and one-way ANOVA analysis for continuous variables

$B M I$ body mass index, TG triglycerides, TC total cholesterol, HDL-C high-density lipoprotein cholesterol, $L D L-C$ low-density lipoprotein cholesterol, apoAl apolipoprotein $\mathrm{Al}, a p o B 100$ apolipoprotein B100

0.05 for all). The ratios of LDL-C/HDL-C and apoB/ apoAI increased, whereas HDL-C and apoAI decreased with the tertile of the Gensini scores $(P<0.05$ for all). There were no significant differences in weight, BMI, prevalence of hypertension and diabetes, TG, TC, LDLC, apoB100, TG/HDL-C and TC/HDL-C across the groups.

The anthropometric and biochemical characteristics of the CHD patients with different number of stenotic vessels are shown in Table 2. The mean age, and the prevalence of male gender, cigarette smoking, hypertension and diabetes increased with the number of stenotic vessels $(P<0.05$ for all). TG, TC, LDL-C, apoB100, the ratios of TG/HDL-C, TC/HDL-C, LDL-C/HDL-C and apoB/apoAI increased, and HDL-C and apoAI decreased with the number of stenotic vessels $(P<0.05$ for all). There were no significant differences in weight and BMI across the groups.

Univariate logistic regression of the lipoprotein ratios and the severity of CHD

Univariate logistic regression analyses showed that the ratio of TG/HDL-C was significantly associated with the number of stenotic vessels $(P<0.05)$, but not with the tertile of the Gensini scores. The ratios of TC/HDL-C, LDL-C/HDL-C and apoB100/apoAI were significantly associated with both the tertile of the Gensini scores and the number of stenotic vessels $(P<0.05$ for all $)$ (Table 3).

\section{Multivariate logistic regression of the lipoprotein ratios and the severity of CHD}

As shown in Table 4, the ratios of TC/HDL-C and LDLC/HDL-C were significantly associated with both the tertile of the Gensini scores and the number of stenotic vessels, and TG/HDL-C was significantly associated with the number of stenotic vessels after adjusting for the non-lipid cardiovascular risk factors including age, gender, weight, BMI, smoking, alcohol consumption, diabetes mellitus and hypertension (model 1). However, all the associations between the ratios of TG/HDL-C, TC/ HDL-C and LDL-C/HDL-C and the severity of CHD became insignificant after adjusting for the variables in model 1 plus the individual lipid variables including TG, HDL-C, LDL-C, apoB100 and apoAI. TC was excluded during the model-building process due to its significant 
Table 2 Clinical characteristics of the CHD patients with different number of stenotic vessels

\begin{tabular}{|c|c|c|c|c|}
\hline Variables & Single-branch stenosis $(n=327)$ & Double-branch stenosis $(n=212)$ & Multi-branch stenosis $(n=253)$ & $P$ value \\
\hline \multicolumn{5}{|l|}{ Non-lipid variables } \\
\hline Age, years & $61.93 \pm 10.25$ & $63.04 \pm 9.56$ & $65.12 \pm 10.25$ & 0.001 \\
\hline Male gender, n (\%) & $197(60.2)$ & $158(74.5)$ & $193(76.3)$ & $<0.001$ \\
\hline Weight, kg & $64.26 \pm 9.62$ & $64.32 \pm 8.71$ & $64.43 \pm 9.81$ & 0.985 \\
\hline $\mathrm{BMl}, \mathrm{kg} / \mathrm{m}^{2}$ & $24.43 \pm 2.80$ & $24.40 \pm 3.15$ & $24.31 \pm 3.13$ & 0.923 \\
\hline Smokers, n (\%) & $119(36.4)$ & $103(48.6)$ & $123(48.6)$ & 0.003 \\
\hline Hypertension, n (\%) & $155(47.4)$ & $97(45.8)$ & $142(56.1)$ & 0.045 \\
\hline Diabetes, n (\%) & $37(11.3)$ & $32(15.1)$ & $51(20.2)$ & 0.013 \\
\hline \multicolumn{5}{|l|}{ Individual lipid variables } \\
\hline $\mathrm{TG}, \mathrm{mmol} / \mathrm{L}$ & $1.44 \pm 0.80$ & $1.63 \pm 1.08$ & $1.67 \pm 1.30$ & 0.049 \\
\hline $\mathrm{TC}, \mathrm{mmol} / \mathrm{L}$ & $4.07 \pm 0.87$ & $4.32 \pm 1.10$ & $4.30 \pm 1.22$ & 0.028 \\
\hline $\mathrm{HDL}-\mathrm{C}, \mathrm{mmol} / \mathrm{L}$ & $1.03 \pm 0.308$ & $0.99 \pm 0.33$ & $0.94 \pm 0.30$ & 0.003 \\
\hline $\mathrm{LDL}-\mathrm{C}, \mathrm{mmol} / \mathrm{L}$ & $2.31 \pm 0.73$ & $2.50 \pm 0.83$ & $2.56 \pm 0.97$ & 0.004 \\
\hline ApoAl, g/L & $1.03 \pm 0.20$ & $1.00 \pm 0.20$ & $0.96 \pm 0.22$ & 0.001 \\
\hline ApoB100, g/L & $0.74 \pm 0.24$ & $0.79 \pm 0.25$ & $0.80 \pm 0.29$ & 0.009 \\
\hline \multicolumn{5}{|l|}{ Lipoprotein ratios } \\
\hline $\mathrm{TG} / \mathrm{HDL}-\mathrm{C}$ & $1.64 \pm 1.36$ & $2.20 \pm 2.70$ & $2.43 \pm 5.05$ & 0.003 \\
\hline $\mathrm{TC} / \mathrm{HDL}-\mathrm{C}$ & $4.26 \pm 1.50$ & $5.10 \pm 4.01$ & $5.12 \pm 3.33$ & $<0.001$ \\
\hline LDL-C/HDL-C & $2.44 \pm 1.03$ & $2.87 \pm 1.83$ & $3.05 \pm 3.05$ & $<0.001$ \\
\hline ApoB/apoAl & $0.74 \pm 0.26$ & $0.81 \pm 0.28$ & $0.88 \pm 0.45$ & $<0.001$ \\
\hline
\end{tabular}

The differences across the groups with different number of stenotic vessels were calculated by Chi-square test for categorical variables, and one-way ANOVA analysis for continuous variables

$B M I$ body mass index, TG triglycerides, TC total cholesterol, HDL-C high-density lipoprotein cholesterol, $L D L-C$ low-density lipoprotein cholesterol, apoAI apolipoprotein Al, apoB100 apolipoprotein B100

collinearity with LDL-C. ApoB100/apoAI was independently and significantly associated with the tertile of the Gensini scores and the number of stenotic vessels after adjusting for the variables in model 2 plus the lipoprotein ratios including TG/HDL-C and LDL-C/HDL-C (Table 4). TC/HDL-C was excluded from the model as it is highly correlated with LDL-C/HDL-C.

\section{Path analysis of the lipoprotein ratios and the severity of CHD}

Path analysis indicated that age, smoking, diabetes, TG, LDL-C, apoAI and apoB100/apoAI had direct effects on the severity of CHD. Among them, age, smoking, apoAI and apoB100/apoAI had direct effect on the tertile of the Gensini scores (Fig. 1), and age, diabetes, TG, LDL$\mathrm{C}$, apoAI and apoB100/apoAI had direct effect on the number of stenotic branches (Fig. 2). The variables including age, male gender, BMI, HDL-C, LDL-C, apoB100 and apoAI also had indirect effects on the severity of CHD mediated by the apoB100/apoAI ratio. There were 11 paths (age $\rightarrow$ apoB100/apoAI $\rightarrow$ the tertile of the Gensini scores or the number of stenotic vessels, BMI $\rightarrow$ apoB100/apoAI $\rightarrow$ the tertile of the Gensini scores or the number of stenotic vessels, HDL-C $\rightarrow$ apoB100/

Table 3 Results of the univariate ordinal logistic regression of the lipoprotein ratios and the tertile of the Gensini scores or the number of stenotic vessels

\begin{tabular}{|c|c|c|c|c|c|c|}
\hline \multirow[t]{2}{*}{ Variables } & \multicolumn{3}{|c|}{ The tertile of the Gensini scores } & \multicolumn{3}{|c|}{ The number of stenotic vessels } \\
\hline & OR & $95 \% \mathrm{Cl}$ & $P$ & OR & $95 \% \mathrm{Cl}$ & $P$ \\
\hline $\mathrm{TG} / \mathrm{HDL}-\mathrm{C}$ & 1.02 & $0.98-1.07$ & 0.341 & 1.05 & $1.01-1.09$ & 0.021 \\
\hline $\mathrm{TC} / \mathrm{HDL}-\mathrm{C}$ & 1.05 & $1.00-1.10$ & 0.034 & 1.09 & $1.04-1.14$ & $<0.001$ \\
\hline LDL-C/HDL-C & 1.12 & $1.02-1.23$ & 0.017 & 1.20 & $1.10-1.31$ & $<0.001$ \\
\hline ApoB100/apoAl & 2.30 & $1.51-3.51$ & $<0.001$ & 3.09 & $2.07-4.61$ & $<0.001$ \\
\hline
\end{tabular}

OR odds ratio, $95 \% \mathrm{Cl} 95 \%$ confidence interval, TG/HDL-C triglyceride/high-density lipoprotein cholesterol, TC/HDL-C total cholesterol/high-density lipoprotein cholesterol, LDL-C/HDL-C low-density lipoprotein cholesterol/high-density lipoprotein cholesterol, apoB100/apoAl apolipoprotein Al/apolipoprotein B100 
Table 4 Results of multivariate ordinal logistic regression of the lipoprotein ratios and the tertile of the Gensini scores or the number of stenotic vessels

\begin{tabular}{|c|c|c|c|c|c|c|}
\hline \multirow[t]{2}{*}{ Variables } & \multicolumn{3}{|c|}{ The tertile of the Gensini scores } & \multicolumn{3}{|c|}{ The number of stenotic vessels } \\
\hline & $\mathrm{OR}$ & $95 \% \mathrm{Cl}$ & $P$ & $\mathrm{OR}$ & $95 \% \mathrm{Cl}$ & $P$ \\
\hline \multicolumn{7}{|l|}{$\mathrm{TG} / \mathrm{HDL}-\mathrm{C}$} \\
\hline Model 1 & 1.02 & $0.98-1.08$ & 0.339 & 1.05 & $1.00-1.10$ & 0.033 \\
\hline Model 2 & 0.98 & $0.91-1.05$ & 0.576 & 1.00 & $0.93-1.07$ & 0.983 \\
\hline Model $3^{\mathrm{a}}$ & 0.98 & $0.91-1.05$ & 0.536 & 1.04 & $0.90-1.21$ & 0.600 \\
\hline \multicolumn{7}{|l|}{ LDL-C/HDL-C } \\
\hline Model 1 & 1.16 & $1.02-1.31$ & 0.019 & 1.39 & $1.22-1.58$ & $<0.001$ \\
\hline Model 2 & 0.99 & $0.827-1.19$ & 0.909 & 1.01 & $0.83-1.22$ & 0.960 \\
\hline Model $3^{b}$ & 1.13 & $0.77-1.66$ & 0.533 & 0.87 & $0.57-1.31$ & 0.499 \\
\hline \multicolumn{7}{|l|}{$\mathrm{TC} / \mathrm{HDL}-\mathrm{C}$} \\
\hline Model 1 & 1.07 & $1.01-1.14$ & 0.030 & 1.13 & $1.06-1.21$ & $<0.001$ \\
\hline Model 2 & 0.97 & $0.89-1.72$ & 0.485 & 0.99 & $0.91-1.08$ & 0.888 \\
\hline Model $3^{b}$ & 0.87 & $0.65-1.16$ & 0.334 & 0.81 & $0.59-1.10$ & 0.176 \\
\hline \multicolumn{7}{|c|}{ ApoB100/apoAl } \\
\hline Model 1 & 2.73 & $1.60-4.66$ & $<0.001$ & 4.79 & $2.80-8.20$ & $<0.001$ \\
\hline Model 2 & 3.18 & $1.30-7.76$ & 0.011 & 2.55 & $1.04-6.28$ & 0.041 \\
\hline Model $3^{c}$ & 2.93 & $1.17-7.34$ & 0.022 & 3.14 & $1.01-6.47$ & 0.048 \\
\hline
\end{tabular}

Model 1, adjusted for the non-lipid CHD risk factors including age, gender, weight, BMI, smoking, alcohol consumption, diabetes mellitus and hypertension; Model 2, adjusted for the variables in Model 1 plus the individual lipid variables including TG, HDL-C, LDL-C, apoB100 and apoAl; Model 3 a , adjusted for the variables in Model 2 plus the lipoprotein ratios including LDL-C/HDL-C and apoB100/apoAl; Model $3^{\mathrm{b}}$, adjusted for the variables in Model 2 plus the lipoprotein ratios including TG/HDL-C and apoB100/apoAl; Model $3^{\mathrm{C}}$, adjusted for the variables in Model 2 plus the lipoprotein ratios including TG/HDL-C and LDL-C/HDL-C OR odds ratio, $95 \%$ Cl $95 \%$ confidence interval, TG/HDL-C triglyceride/high-density lipoprotein cholesterol, $T C / H D L-C$ total cholesterol/high-density lipoprotein cholesterol, $L D L-C / H D L-C$ low-density lipoprotein cholesterol/high-density lipoprotein cholesterol, apoB100/apoAl apolipoprotein $\mathrm{Al} / \mathrm{apolipoprotein} \mathrm{B} 100$

apoAI $\rightarrow$ the tertile of the Gensini scores or the number of stenotic vessels, age $\rightarrow \mathrm{HDL}-\mathrm{C} \rightarrow$ apoB100/apoAI $\rightarrow$ the tertile of the Gensini scores or the number of stenotic vessels, male gender $\rightarrow \mathrm{HDL}-\mathrm{C} \rightarrow$ apoB100/ apoAI $\rightarrow$ the tertile of the Gensini scores or the number of stenotic vessels, BMI $\rightarrow$ HDL-C $\rightarrow$ apoB100/apoAI $\rightarrow$ the tertile of the Gensini scores or the number of stenotic vessels, LDL-C $\rightarrow$ apoB100/apoAI $\rightarrow$ the tertile of the Gensini scores or the number of stenotic vessels, $\mathrm{BMI} \rightarrow \mathrm{LDL}-\mathrm{C} \rightarrow$ apoB100/apoAI $\rightarrow$ the tertile of the Gensini scores or the number of stenotic vessels, apoAI $\rightarrow$ apoB100/apoAI $\rightarrow$ the tertile of the Gensini

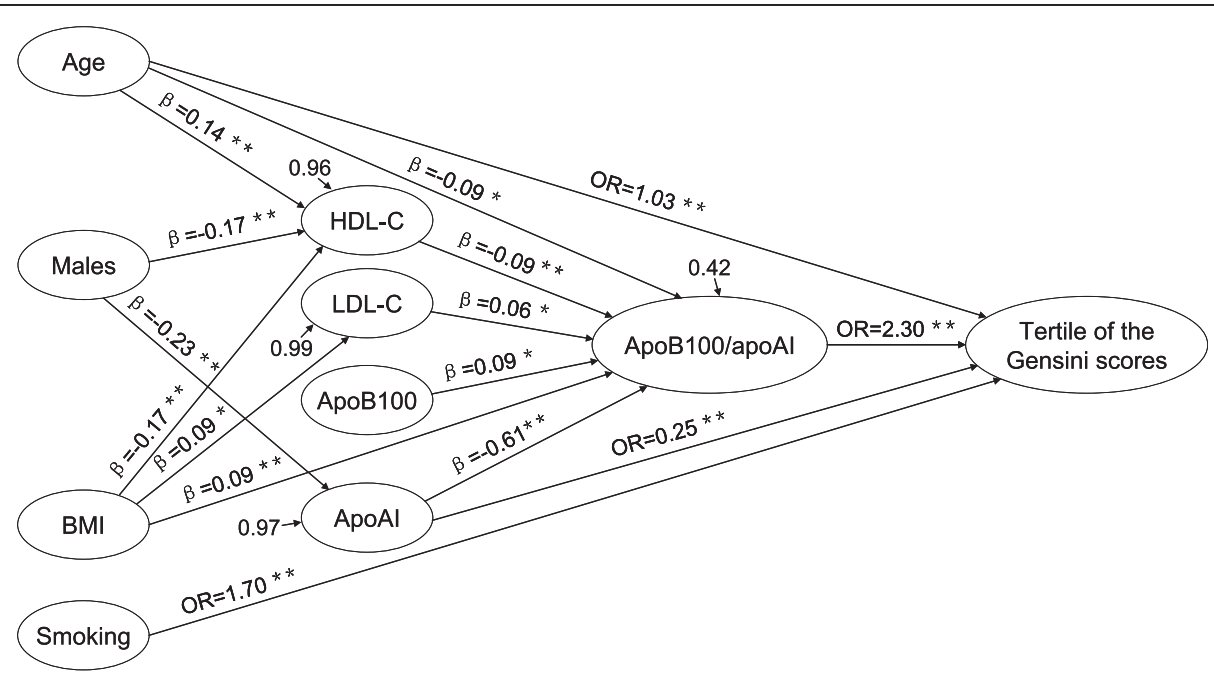

Fig. 1 Significant regression paths among dependent (the tertile of the Gensini scores) and independent variables. ${ }^{*} P \leq 0.05,{ }^{* *} P \leq 0.01$, standardized regression coefficients ( $\beta$ ) or odds ratios (OR) represented as one-way arrows 


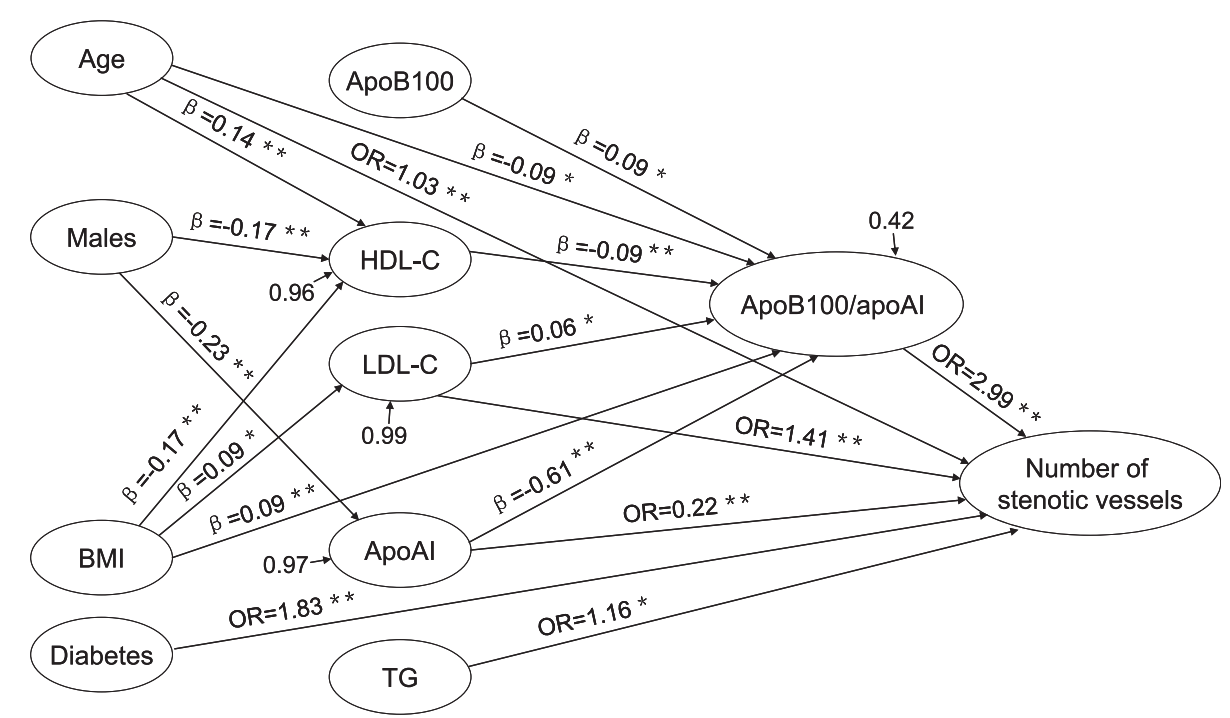

Fig. 2 Significant regression paths among dependent (the number of stenotic vessels) and independent variables. ${ }^{*} P \leq 0.05,{ }^{* *} P \leq 0.01$, standardized regression coefficients ( $\beta$ ) or odds ratios (OR) represented as one-way arrows

scores or the number of stenotic vessels, male gender $\rightarrow$ apoAI $\rightarrow$ apoB100/apoAI $\rightarrow$ the tertile of the Gensini scores or the number of stenotic vessels, and apoB100 $\rightarrow$ apoB100/apoAI $\rightarrow$ the tertile of the Gensini scores or the number of stenotic vessels) through which apoB100/ apoAI mediated the indirect effects of the CHD risk factors on the severity of CHD.

\section{Discussion}

We have previously established a predictive role of lipoprotein ratios for the occurrence of CHD [7]. We found that the lipoprotein ratios were superior to individual lipid parameters as predictors for CHD, and apoB100/ apoAI was the best one to predict the CHD risk. In the present study, the associations between the lipoprotein ratios and the severity of CHD were further investigated and the results showed that the ratio of apoB100/apoAI, but not the HDL-C related ratios (i.e. TG/HDL-C, TC/ HDL-C and LDL-C/HDL-C), was significantly and independently associated with the severity of CHD. The statistical power calculations revealed that there was $\geq 87 \%$ power to detect a significant association between the ratio of apoB100/apoAI and the severity of CHD $(\alpha=0.05)$ with an effect size of ORs $\geq 2.30$ in univariate and multivariate analyses. The design of our study is unique in that both the Gensini scores and the number of stenotic coronary branches were employed to evaluate the severity of CHD in one study.

To the best of our knowledge, this study is the first one indicating that the apoB100/apoAI ratio is a strong predictor for the severity of CHD in Chinese Han people. The Han people are the largest ethnic group in East Asia, constituting approximately $92 \%$ of the population of Mainland China, $98 \%$ of the population of Taiwan, and $74 \%$ of the population of Singapore. Previous studies have shown that high apoB100/apoAI ratio is independently correlated with the presence of angiographic CHD [7, 13, 17, 18]. In the current study, we further revealed that this ratio index is independently associated with the severity of CHD. The apoB100/apoAI ratio increased orderly with the severity of stenosis expressed by the tertile of the Gensini scores (Table 1), but also with the number of stenotic coronary branches (Table 2). In univariate logistic regression, the apoB100/apoAI ratio showed a strong relationship with the tertile of the Gensini scores and the number of stenotic vessels (Table 3), and this association was not appreciably altered after adjusting for age, gender, weight, BMI, smoking, alcohol consumption, diabetes mellitus, hypertension, TG, LDL-C, HDL-C, TG/HDL-C and LDL-C/HDL-C in the following multivariate logistic regression (Table 4). Taken together, these results indicate that the apoB100/apoAI ratio could be a strong predictor for the severity of CHD.

In path analysis, we found that some conventional cardiovascular risk factors such as male gender, BMI, HDLC, LDL-C, apoB100 and apoAI had indirect effects on the severity of CHD, and apoB100/apoAI acted as a key mediator in this process. Age and apoAI can directly affect the severity of CHD, and they also had indirect effects on the severity of $\mathrm{CHD}$ through the mediation of apoB100/apoAI. In addition, the results of the path analysis showed that smoking and diabetes had significant effects on the severity of CHD independently of the ratio of apoB100/apoAI and other lipid parameters. 
It is theoretically reasonable that the ratio of apoB100/ apoAI is strongly associated with the severity of CHD. Each particle of the atherogenic lipoproteins, such as low-density lipoprotein, very low-density lipoprotein, intermediate-density lipoprotein and lipoprotein(a), carries one apoB100 molecule, so the concentrations of plasma apoB100 can reflect the total of proatherogenic potentials. On the other hand, apoAI is the major apolipoprotein component of antiatherogenic lipoprotein, i.e. high-density lipoprotein, the serum content of apoAI represents the total of antiatherogenic potentials. Thus, the apoB100/apoAI ratio could comprehensively reflect the balance between the atherogenic and antiatherogenic potentials in one individual. The higher is the apoB100/ apoAI ratio, the more are the atherogenic potentials, or the less are the antiatherogenic potentials, or both.

The ratio of TG/HDL-C was found to be associated with the number of stenotic vessels, but not the tertile of the Gensini scores by univariate logistic regression (Table 3). In multivariate logistic regression, TG/HDL-C was significantly associated with the number of stenotic vessels after adjusting for the non-lipid cardiovascular risk factors including age, gender, weight, BMI, smoking, alcohol consumption, diabetes mellitus and hypertension in model 1 . However, it became insignificant when the individual lipid parameters (i.e. TG, LDL-C, HDL-C, apoB100 and apoAI) were added to the model (model 2 ), which indicates that the predictive power of TG/ HDL-C to the number of stenotic vessels can be replaced by the individual lipid parameters. Our findings are consistent with the results of a hospital-based study [9], in which Yang D et al. demonstrated that the TG/ HDL-C ratio was not associated with the Gensini scores in Chinese CHD patients. However, in another casecontrol study which was also involved in Chinese CHD patients, Yunke $\mathrm{Z}$ et al. [11] reported that the TG/HDL$\mathrm{C}$ ratio can predict the severity of CHD and the incident of new-onset heart failure among in-hospital CHD patients. In Brazilians, da Luz PL et al. [12] also demonstrated that the TG/HDL-C ratio was associated with the severity of CHD. The association between the TG/HDL$\mathrm{C}$ ratio and the severity of $\mathrm{CHD}$ needs to be further studied.

The ratios of TC/HDL-C and LDL-C/HDL-C were associated with both the tertile of the Gensini scores and the number of stenotic vessels in univariate logistic regression analyses (Table 3). In the following multivariate logistic regression, the significant associations persisted when controlled for the non-lipid variables in model 1 . However, the associations were completely abolished for both TC/HDL-C and LDL-C/HDL-C when individual lipid variables were added in model 2 . The associations between the ratios of TC/HDL-C and LDL-C/HDL-C and the severity of $\mathrm{CHD}$ could be mediated by the individual lipid parameters. Contrary to our findings, the significant associations between the ratios of TC/HDL-C and LDL-C/HDL-C and the severity of CHD were reported by other investigators. Yang D et al. [9] reported a significant and positive correlation between the ratios of TC/HDL-C and LDL/HDL-C and the Gensini scores in Chinese patients. Lippi $\mathrm{U}$ et al. [8] demonstrated that the TC/HDL-C and LDL/HDL-C ratios, but not the plasma levels of TG, TC, HDL-C and LDL-C are statistically related to the mild severity of $\mathrm{CHD}$. In a recent cross-sectional association study [10], Platt DE et al. reported that the TC/HDL-C ratio is a strong biological marker for CHD occurrence and severity, and the TC/ HDL-C ratio $\geq 4$ significantly predicts $\geq 50 \%$ stenosis in all vessels. However, apoB100 and apoAI were not enrolled into the regression models in all the abovementioned studies [8-10], so it is not clear that the associations between the TC/HDL-C and LDL/HDL-C ratios and the severity of $\mathrm{CHD}$ were independent of apoB100 and apoAI.

Our study has several limitations. First, the present study was not designed to examine all the risk factors associated with the severity of CHD. Rather, the specific goal was to examine whether the lipoprotein ratios were associated with the severity of CHD. Second, our study mainly focused on the CHD patients whose lesions of coronary artery were $50 \%$ or more in at least one major branch according to the diagnostic criteria. Those with mild coronary stenosis (less than $50 \%$ ) were not enrolled in this study although the lipoprotein ratios might also be associated with mild stenosis.

\section{Conclusions}

In conclusion, the results of our study suggest that the ratio of apoB100/apoAI could be a powerful predictor for the severity of CHD.

\section{Patients and methods CHD patients}

Details of this study have been published previously [7]. Briefly, a total of 792 consecutive unrelated atherosclerotic CHD patients who underwent coronary angiography at the Affiliated Hospital of North Sichuan Medical College (Nanchong, China) were enrolled in the study between May 2011 and April 2015. Patients taking lipid lowering drugs or the drugs that might affect the glucose or lipid metabolism were excluded from the study. In order to enlarge the sample size, the patients who took the drugs which were thought not to affect plasma lipid levels were still enrolled in the study. Patients with renal or hepatic dysfunction, significant valvular disease, myocarditis, and malignant disease were excluded from the study. All the subjects were Chinese Han people. The tenets of the Declaration of Helsinki were adhered to in 
all procedures in the study. The study protocol was approved by the ethics committee of North Sichuan Medical College. Written consent was provided by all the participants or their guardians prior to their participation in the study.

\section{Coronary angiography}

The Gensini scoring system and the number of stenotic coronary branches were used to assess the severity of CHD. Patients were stratified into three groups based on the tertile of the Gensini scores or the number of stenotic coronary branches. Coronary angiograms were evaluated by experienced cardiologists who were not aware of the patients' biochemical status. Standard coronary angiography with at least two views of the right coronary artery and four views of the left coronary system were performed using the Judkins technique by Allura Xper FD20 (Philips Medical Systems Nederland B.V. Netherlands). Atherosclerotic CHD was diagnosed in patients who had angiographic evidence of stenosis greater than $50 \%$ in at least one major coronary artery. Those with normal coronary arteries or minimal stenosis (less than $50 \%$ ) in any of the major coronary arteries were excluded from the study.

\section{Diagnostic criteria}

Body mass index (BMI) was calculated by dividing weight by height squared $\left(\mathrm{kg} / \mathrm{m}^{2}\right)$. Smoking was defined as regular cigarette smoking. Hypertension was defined as the measurement of systolic/diastolic blood pressure higher than 140/90 mmHg or active use of antihypertensive drugs. Diabetes mellitus was defined as the fasting glucose levels above $126 \mathrm{mg} / \mathrm{dL}$ or active use of antidiabetic drugs or insulin.

\section{Biochemical measurement}

Fasting blood samples were taken on the first morning of the in-hospital day when lipid-lowering drugs were not yet used. Samples were immediately transported to the Department of Clinical Laboratory of the Affiliated Hospital of North Sichuan Medical College for measurement of plasma lipids. TG, TC, LDL-C and HDL-C were measured directly by enzymatic methods. ApoB100 and apoAI were measured by immunoturbidimetric assays. All the measurements were carried out using an automatic clinical chemistry analyzer (Beckman Coulter AU5800, USA). Lipoprotein ratios were calculated.

\section{Statistical analysis}

Data are presented as mean \pm standard deviation (SD) unless otherwise stated. Continuous variables were tested for normality; otherwise, log transformation was applied. All statistical analyses were carried out by using SPSS version 13.0 (SPSS Inc., Chicago, IL, USA). The differences among the three groups categorized by the tertile of the Gensini scores or the number of stenotic vessels were calculated by Chi-square test for categorical variables, and one-way ANOVA analysis for continuous variables. Univariate and multivariate ordinal logistic regression analyses were employed to determine the associations between the lipoprotein ratios and the severity of atherosclerotic CHD. The results of logistic regression analysis were expressed as the odds ratio (OR) with $95 \%$ confidence interval $(95 \% \mathrm{CI})$. In the multivariate regression, collinearity among the independent variables was diagnosed by examining variance inflation factor (VIF) and tolerance. Path analysis was used to examine the causal relationships among the non-lipid cardiovascular risk factors, individual lipid variables, lipoprotein ratios and the severity of CHD. Power calculations were performed using the PASS 14 program based on Hsieh's method [19]. All $P$-values are two-tailed and differences were considered significant if $P \leq 0.05$.

\section{Abbreviations}

CHD: Coronary heart disease; BMl: Body mass index; TG: Triglycerides; TC: Total cholesterol; HDL-C: High-density lipoprotein cholesterol; LDL-C: Low-density lipoprotein cholesterol; ApoB100: Apolipoprotein B100; ApoAl: Apolipoprotein Al.

\section{Competing interests}

The authors declare that they have no competing interests.

\section{Authors' contributions}

YYS and ZL conceived of the study, participated in the design, and drafted the manuscript. ZL and XMZ conducted the coronary angiography. YYS and JXZ performed the statistical analysis. JXZ, YMW, YY, WFH, and JZ collected the data. All authors read and approved the final manuscript.

\section{Acknowledgements}

This research was supported by the grants from the Basic and Applied Research Project of Sichuan Province, China (2013JY0072) and the Key Cultivation Project of North Sichuan Medical College, Sichuan Province, China (CBY12-A-ZP06).

\section{Author details}

'Department of Medical Biochemistry, School of Preclinical Medicine, North Sichuan Medical College, Nanchong 637000, P. R. China. ${ }^{2}$ School of Clinical Medicine, North Sichuan Medical College, Nanchong 637000, P. R. China. ${ }^{3}$ Department of Cardiology, Affiliated Hospital of North Sichuan Medical College, Nanchong 637000, Sichuan Province, P. R. China. ${ }^{4}$ College of Medicine, Yaan Vocational and Technical College, Yaan 625000, P. R. China. ${ }^{5}$ Sichuan Key Laboratory of Medical Imaging, and Department of Radiology, Affiliated Hospital of North Sichuan Medical College, Nanchong 637000, Sichuan Province, China.

Received: 2 September 2015 Accepted: 12 November 2015 Published online: 18 November 2015

\section{References}

1. Bi $Y$, Jiang $Y$, He J, Xu $Y$, Wang $L, X u M$, et al. Status of cardiovascular health in Chinese adults. J Am Coll Cardiol. 2015;65:1013-25.

2. Yusuf S, Hawken S, Ounpuu S, Dans T, Avezum A, Lanas F, et al. Effect of potentially modifiable risk factors associated with myocardial infarction in 52 countries (the INTERHEART study): case-control study. Lancet. 2004:364:937-52.

3. Stone NJ, Robinson JG, Lichtenstein AH, Bairey Merz CN, Blum CB, Eckel RH, et al. 2013 ACC/AHA guideline on the treatment of blood cholesterol to reduce atherosclerotic cardiovascular risk in adults: a report of the American 
College of Cardiology/American Heart Association Task Force on Practice Guidelines. Circulation. 2014;129:S1-45.

4. National Cholesterol Education Program (NCEP) Expert Panel on Detection, Evaluation, and Treatment of High Blood Cholesterol in Adults (Adult Treatment Panel III). Third Report of the National Cholesterol Education Program (NCEP) Expert Panel on Detection, Evaluation, and Treatment of High Blood Cholesterol in Adults (Adult Treatment Panel III) final report. Circulation. 2002;106:3143-421.

5. Hsia SH, Pan D, Berookim P, Lee ML. A population-based, cross-sectional comparison of lipid-related indexes for symptoms of atherosclerotic disease. Am J Cardiol. 2006;98:1047-52.

6. Kastelein JJ, van der Steeg WA, Holme I, Gaffney M, Cater NB, Barter P, et al. Lipids, apolipoproteins, and their ratios in relation to cardiovascular events with statin treatment. Circulation. 2008;117:3002-9.

7. Zhu L, Lu Z, Zhu L, Ouyang X, Yang Y, He W, et al. Lipoprotein ratios are better than conventional lipid parameters in predicting coronary heart disease in Chinese Han people. Kardiol Pol. 2015:73:723-30

8. Lippi U, Cappelletti P, Signori D, Burelli C. Clinical chemical indexes and severity of coronary atherosclerosis. Clin Chim Acta. 1983;130:283-9.

9. Yang D, Liu X, Xiang M. The correlation between lipids ratio and degree of coronary artery stenosis. High Blood Press Cardiovasc Prev. 2011;18:53-6.

10. Platt DE, Ghassibe-Sabbagh M, Youhanna S, Hager J, Cazier JB, Kamatani Y, et al. Circulating lipid levels and risk of coronary artery disease in a large group of patients undergoing coronary angiography. J Thromb Thrombolysis. 2015;39:15-22.

11. Yunke Z, Guoping L, Zhenyue C. Triglyceride-to-HDL cholesterol ratio. Predictive value for $\mathrm{CHD}$ severity and new-onset heart failure. Herz. 2014:39:105-10.

12. da Luz PL, Favarato D, Faria-Neto Jr JR, Lemos P, Chagas AC. High ratio of triglycerides to HDL-cholesterol predicts extensive coronary disease. Clinics (Sao Paulo). 2008;63:427-32.

13. Walldius G, Jungner I, Aastveit AH, Holme I, Furberg CD, Sniderman AD. The apoB/apoA-I ratio is better than the cholesterol ratios to estimate the balance between plasma proatherogenic and antiatherogenic lipoproteins and to predict coronary risk. Clin Chem Lab Med. 2004;42:1355-63.

14. Liem AH, van de Woestijne AP, van Lennep HW R, Zwinderman AH, van der Steeg WA, Jukema JW. ApoB/A1 and LDL-C/HDL-C and the prediction of cardiovascular risk in statin-treated patients. Curr Med Res Opin. 2008;24:359-64.

15. Goswami B, Rajappa M, Mallika V, Kumar S, Shukla DK. Apo-B/apo-Al ratio: a better discriminator of coronary artery disease risk than other conventional lipid ratios inlndian patients with acute myocardial infarction. Acta Cardiol. 2008:63:749-55.

16. McQueen MJ, Hawken S, Wang X, Ounpuu S, Sniderman A, Probstfield J, et al. Lipids, lipoproteins, and apolipoproteins as risk markers of myocardial infarction in 52 countries (thelNTERHEART study): a case-control study. Lancet. 2008;372:224-33.

17. Tamang HK, Timilsina U, Singh KP, Shrestha S, Raman RK, Panta P, et al. Apo B/Apo A-I Ratio is Statistically A Better Predictor of Cardiovascular Disease (CVD) than Conventional LipidProfile: A Study from Kathmandu Valley, Nepal. J Clin Diagn Res. 2014;8:34-6.

18. Pan L, Lu G, Chen Z. Combined use of apolipoprotein B/apolipoprotein A1 ratio and non-high-density lipoprotein cholesterol before routine clinical lipid measurement in predicting coronary heart disease. Coron Artery Dis. 2014;25:433-8

19. Hsieh FY, Bloch DA, Larsen MD. A simple method of sample size calculation for linear and logistic regression. Stat Med. 1998;17:1623-34.

\section{Submit your next manuscript to BioMed Central and take full advantage of:}

- Convenient online submission

- Thorough peer review

- No space constraints or color figure charges

- Immediate publication on acceptance

- Inclusion in PubMed, CAS, Scopus and Google Scholar

- Research which is freely available for redistribution

Submit your manuscript at www.biomedcentral.com/submit 